\title{
Toosendanin induces the apoptosis of human Ewing's sarcoma cells via the mitochondrial apoptotic pathway
}

\author{
TIAN GAO*, AN XIE*, XUQIANG LIU*, HAIBO ZHAN, JIN ZENG, MIN DAI and BIN ZHANG \\ Department of Orthopedics, Multidisciplinary Therapy Center of Musculoskeletal Tumor, \\ The First Affiliated Hospital of Nanchang University, Nanchang, Jiangxi 330006, P.R. China
}

Received October 15, 2018; Accepted April 26, 2019

DOI: $10.3892 / \mathrm{mmr} .2019 .10224$

\begin{abstract}
Toosendanin, a triterpenoid extracted from the root bark of Melia toosendan, has its origin from traditional Chinese medicine and has been used as a non-polluting and pesticide-free plant insecticide in China for fruit and vegetable production. In recent years, toosendanin has been found to inhibit tumor cell proliferation and promote tumor cell apoptosis. Ewing's sarcoma (ES) is the second most common primary malignant bone and soft tissue tumor in children and adolescents. Although the overall prognosis of ES has improved, the 5-year survival rate has not significantly increased. To analyze the role of toosendanin on ES progression, CCK-8 viability assay, flow cytometry, Hoechst 33258 staining and western blotting were performed. The present results suggested that toosendanin suppressed cell viability and induced apoptosis in human SK-ES-1 cells compared with DMSO treatment. In addition, in the present study, toosendanin was found to upregulate the expression of Bax and downregulate the expression of $\mathrm{Bcl}-2$, altering the $\mathrm{Bax} / \mathrm{Bcl}-2$ ratio. Additionally, toosendanin promoted the release of cytochrome $\mathrm{c}$, resulting in the activation of the mitochondrial apoptotic pathway, thus inducing the activation of caspase- 9 and caspase- 3 , and the cleavage of PARP. Our results demonstrated that toosendanin inhibited the growth of ES cells in a dose-dependent manner and triggered mitochondrial apoptotic pathway to induce apoptosis. Therefore, toosendanin can potentially be utilized as an anticancer botanical drug for the treatment of ES.
\end{abstract}

Correspondence to: Dr Min Dai or Dr Bin Zhang, Department of Orthopedics, Multidisciplinary Therapy Center of Musculoskeletal Tumor, The First Affiliated Hospital of Nanchang University, 17 Yongwai Street, Nanchang, Jiangxi 330006, P.R. China

E-mail: w6812039@126.com

E-mail: 13687028270@163.com

${ }^{*}$ Contributed equally

Abbreviations: TCM, traditional Chinese medicine; ES, Ewing's sarcoma; Bcl-2, B-cell lymphoma; Bax, Bcl-2-associated X protein; PARP, poly(ADP-ribose) polymerase; FBS, fetal bovine serum

Key words: anticancer botanical drug, apoptosis, human Ewing's sarcoma, invasion, migration, toosendanin

\section{Introduction}

Traditional Chinese medicine (TCM) has been gaining more attention due to its satisfactory clinical results and the reduced side effects when utilized for cancer treatment. TCM is an important part of complementary and alternative medicines as it has standardized diagnostic and therapeutic systems and is implemented worldwide (1). There is increasing evidence that various herbs and compounds derived from natural products with antitumor effects can induce apoptosis in various tumor cells (2-4). Toosendanin is a triterpenoid extracted from the root bark of Melia toosendan. Toosendanin was found to inhibit tumor cell proliferation and promote tumor cell apoptosis (5-7). In addition, a previous study showed that toosendanin can block osteosarcoma tumorigenesis (8). However, there has been no research on the effects of toosendanin on Ewing's sarcoma (ES).

ES is a rare invasive tumor in the primitive neuroectodermal tumor family (9), which is common in children and adolescents $(10,11)$. ES is extremely malignant, with a short disease course, rapid recurrence and high transfer rate. Although neoadjuvant chemotherapy and limb salvage surgery are widely used clinically, the 5-year survival rate for ES patients with early metastasis or recurrence is still less than $50 \%$ and the 10 -year survival rate is less than $30 \%(12,13)$. In addition, most neoadjuvant chemotherapy drugs have the risk of unstable effectiveness and serious side effects such as cardiotoxicity and nephrotoxicity. Therefore, there is an urgent need for safer and more effective anticancer drugs in clinical practice.

Studies have shown that baicalein extracted from the TCM Astragalus membranaceus can induce apoptosis through the mitochondrial apoptosis and death receptor pathways in ES cells (14). Therefore, we hypothesize that toosendanin may also have antitumor and/or pro-apoptotic activity in ES. Subsequently, we studied the effects of toosendanin on cell activity and apoptosis of the human ES cell line SK-ES-1 and further elucidated the relevant molecular mechanisms.

\section{Materials and methods}

Cell culture. The human ES cell lines SK-ES-1 and RD-ES were obtained from the American Type Culture Collection (ATCC; Manassas, VA, USA). The cells were cultured in 
RPMI-1640 medium supplemented with $10 \%$ (v/v) fetal bovine serum (FBS), $100 \mathrm{U} / \mathrm{ml}$ penicillin and $100 \mu \mathrm{g} / \mathrm{ml}$ streptomycin. The cells were incubated at $37^{\circ} \mathrm{C}$ in a humidified atmosphere containing $5 \% \mathrm{CO}_{2}$. Cells were passaged when they reached $80 \%$ confluency. Exponential-phase cells were used in the experiments, and the passage number was $<20$.

Reagents and antibodies. Purified toosendanin (source: root bark and bark of Melia toosendan; molecular formula (MF): $\mathrm{C}_{30} \mathrm{H}_{38} \mathrm{O}_{11}$; molecular weight (MW): 574.62; purity $\geq 98 \%$ ) was purchased from Sigma-Aldrich (Merck KGaA, Darmstadt, Germany). RPMI-1640 medium, phosphate-buffered saline (PBS), dimethyl sulfoxide (DMSO), bovine serum albumin (BSA), Cell Counting Kit-8 (CCK-8) and horseradish peroxidase-conjugated goat anti-rabbit secondary antibodies (dilution 1:2,500; cat. no. HS101) were purchased from TransGen Biotech, Inc. (Beijing, China). FBS was obtained from HyClone Laboratories (GE Healthcare, Chicago, IL, USA), while the Annexin V-FITC/Propidium Iodide (PI) Apoptosis Assay kit was obtained from Becton Dickinson (BD Biosciences, San Jose, CA, USA). The Hoechst 33258 staining kit was purchased from Nanjing KeyGen Biotech Co., Ltd. (Nanjing, China). Antibodies against B-cell lymphoma 2 (Bcl-2; dilution 1:2,000; cat. no. ab182858), Bcl-2-associated X protein (Bax; dilution 1:1,000; cat. no. ab32503), cytochrome $c$ (dilution 1:5,000; cat. no. ab133504), caspase-3 (dilution 1:5,000; cat. no. ab32351), caspase-8 (dilution 1:1,000; cat. no. ab108333), caspase-9 (dilution 1:1,000; cat. no. ab32539), poly(ADP-ribose) polymerase (PARP; dilution 1:1,000; cat. no. ab32138) and GAPDH (dilution 1:2,500; cat. no. ab9485) were purchased from Abcam (Cambridge, UK).

Determination of cell viability by the CCK-8 method. SK-ES-1 and RD-ES cells were cultured in 96-well plates $\left(5 \times 10^{3}\right.$ cells/well). Cells were treated with different concentrations $(0,1,2,5,10,20,40,50$ and $60 \mu \mathrm{M})$ of toosendanin for 24,48 and $72 \mathrm{~h}$ and control cells were treated with $<0.1 \%(\mathrm{v} / \mathrm{v})$ DMSO. After the indicated incubation times, $10 \mu \mathrm{l}$ of CCK-8 was added to the plates and incubated for an additional 1-4 $\mathrm{h}$ at $37^{\circ} \mathrm{C}$. Thereafter, the absorbance was measured at $450 \mathrm{~nm}$ using an ELISA plate reader (ELx800; BioTek Instruments, Inc., Winooski, VT, USA).

Hoechst 33258 nuclear staining. Cells $\left(5 \times 10^{4}\right.$ cells/well) were incubated with 0,25 or $50 \mu \mathrm{M}$ toosendanin in 24-well plates for $24 \mathrm{~h}$ at $37^{\circ} \mathrm{C}$. The cells were then fixed with $4 \%$ paraformaldehyde for $30 \mathrm{~min}$. Thereafter, the cells were washed three times with pre-cooled PBS and stained with $10 \mathrm{mg} / \mathrm{l}$ Hoechst 33258 solution for $10 \mathrm{~min}$ at $25^{\circ} \mathrm{C}$ in the dark. Subsequently, the stained nuclei were observed under a fluorescence microscope (Olympus Corp., Tokyo, Japan) at $350 \mathrm{~nm}$ excitation and $460 \mathrm{~nm}$ emission wavelengths (magnification, $\mathrm{x} 200$ ).

Annexin V-FITC/PI apoptosis assay. SK-ES-1 cells were cultured for $24 \mathrm{~h}$ with 0,25 , or $50 \mu \mathrm{M}$ toosendanin, washed twice with ice-cold PBS, and resuspended at a concentration of $1 \times 10^{6}$ cells $/ \mathrm{ml}$ in $1 \mathrm{X}$ binding buffer. The cell suspension (100 $\mu \mathrm{l})$ was incubated with $1 \mu \mathrm{l}$ Annexin V-FITC and $2 \mu \mathrm{l}$ propidium iodine (PI) solution for $15 \mathrm{~min}$ at $25^{\circ} \mathrm{C}$ in the dark. After addition of $150 \mu \mathrm{l} 1 \mathrm{X}$ binding buffer, the samples were analyzed using a FACSVerse flow cytometer (BD Biosciences, San Jose, CA, USA). Apoptosis rates were analyzed using FlowJo v7.6 software (Tree Star, Inc., Ashland, OR, USA).

Western blot analysis. SK-ES-1 cells were cultured in a 6-well plate at a density of $2 \times 10^{5}$ cells/well. After treatment with 0 , 25 or $50 \mu \mathrm{M}$ toosendanin for $24 \mathrm{~h}$, cells were harvested and lysed in RIPA buffer containing a protease inhibitor cocktail (Sigma-Aldrich; Merck KGaA). The lysate was centrifuged at $12,000 \times \mathrm{g}$ for $10 \mathrm{~min}$ at $4^{\circ} \mathrm{C}$. The supernatant was then collected, and the protein concentration was determined by the BCA method. The same protein amounts $(10 \mu \mathrm{g}$ in each lane) were loaded and separated by $10 \%$ SDS-PAGE, followed by transfer onto polyvinylidene difluoride (PVDF) membranes. The membranes were blocked with $5 \%(\mathrm{w} / \mathrm{v})$ fat-free milk in Tris-buffered saline containing $0.05 \%$ Tween-20 (TBS-T) and then incubated with primary antibodies at $4^{\circ} \mathrm{C}$ overnight. The next day, the PVDF membranes were washed three times in TBS-T and incubated with HRP-conjugated secondary antibodies for $2 \mathrm{~h}$ at room temperature. Immunoreactive proteins were detected by an ECL kit (Thermo Fisher Scientific, Inc.) and then developed on an X-ray film (Kodak). The proteins were quantified via densitometry using ImageJ software (version 1.51j81; National Institutes of Health).

Statistical analysis. Data are expressed as mean \pm standard deviation (SD) and analyzed by GraphPad Prism 7.0 software (GraphPad Software, Inc., Chicago, IL, USA). One-way analysis of variance (ANOVA) was conducted with the Newman-Keuls method to determine the significance of the differences between the experimental conditions. All experiments were repeated at least three times. Differences in means were considered statistically significant at ${ }^{*} \mathrm{P}<0.05,{ }^{* *} \mathrm{P}<0.01$ and ${ }^{* * *} \mathrm{P}<0.001$ (as indicated in the figure legends).

\section{Results}

Toosendanin inhibits cell growth of SK-ES-1 and RD-ES cells. To study the effect of toosendanin on ES cell activity, SK-ES-1 and RD-ES cells were exposed to different concentrations of toosendanin for $24 \mathrm{~h}$. CCK- 8 results demonstrated that toosendanin inhibited the viability of SK-ES-1 and RD-ES cells in a dose-dependent manner and that SK-ES-1 cells were more sensitive to toosendanin (Fig. 1A). The half maximal inhibitory concentration $\left(\mathrm{IC}_{50}\right)$ of SK-ES-1 cells treated with toosendanin was $32.95 \mu \mathrm{M}$ at $24 \mathrm{~h}$. Furthermore, it was observed that toosendanin inhibited the viability of SK-ES-1 in a time- and dose-dependent manner (Fig. 1B). Subsequently, SK-ES-1 cells were treated with toosendanin at concentrations of 0,25 and $50 \mu \mathrm{M}$ for $24 \mathrm{~h}$ in the following assays.

Toosendanin results in morphological changes in SK-ES-1 cells. SK-ES-1 cells treated with toosendanin and stained with Hoechst 33258 showed concentrated and broken nuclei in a dose-dependent manner, which are typical morphological features of apoptotic cells (Fig. 2A).

Toosendanin induces apoptosis. Apoptosis was measured by flow cytometry with Annexin V-FITC/PI double labeling. The apoptosis rate of the control group (sum of early and late 
A

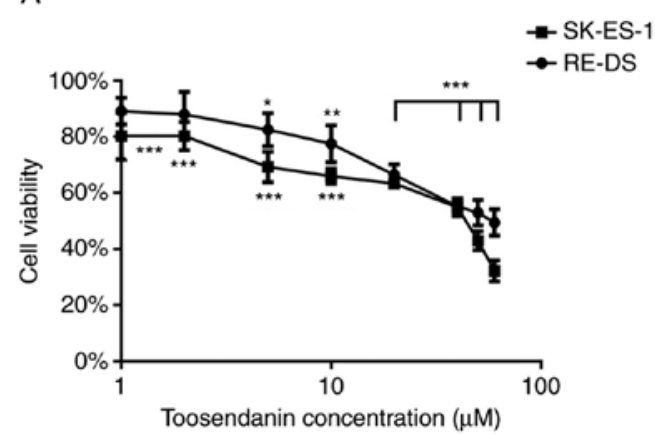

B

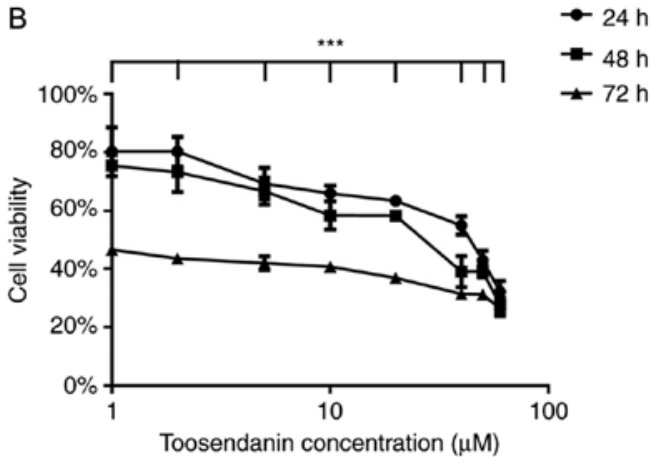

Figure 1. (A) SK-ES-1 cells and RD-ES cells were treated with different concentrations of toosendanin $(0,1,2,5,10,20,40,50$ and $60 \mu \mathrm{M})$ for $24 \mathrm{~h}$ and a CCK-8 assay was performed to detect cell viability after treatment. Data are expressed as the mean $\pm \mathrm{SD}$ of three independent experiments. ${ }^{*} \mathrm{P}<0.05,{ }^{* *} \mathrm{P}<0.01$ and ${ }^{* * * *} \mathrm{P}<0.001$ vs. the control. (B) SK-ES-1 cells were treated with different concentrations of toosendanin $(0,1,2,5,10,20,40,50$ and $60 \mu \mathrm{M})$ for 24,36 , and $48 \mathrm{~h}$ and then a CCK-8 assay was performed to detect cell viability after treatment. Data are expressed as the mean \pm SD of three independent experiments. ${ }^{* * *} \mathrm{P}<0.001$ vs. the control.

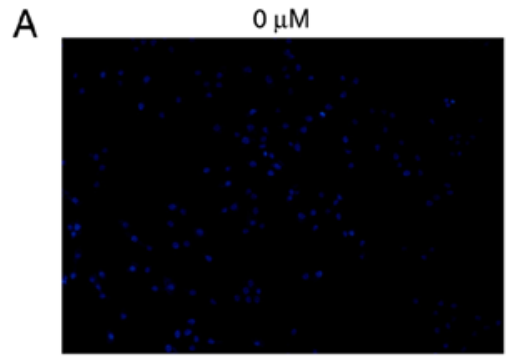

B

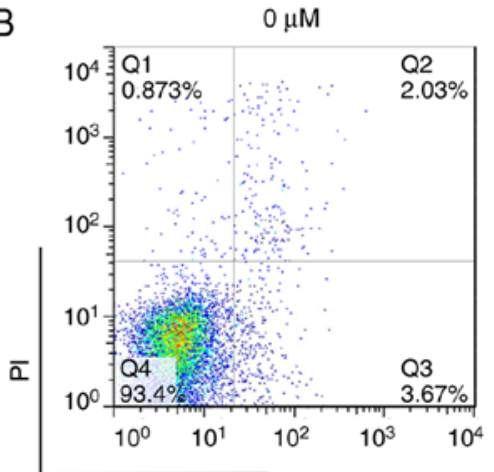

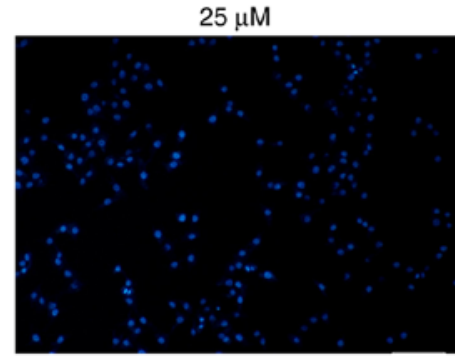

$25 \mu \mathrm{M}$

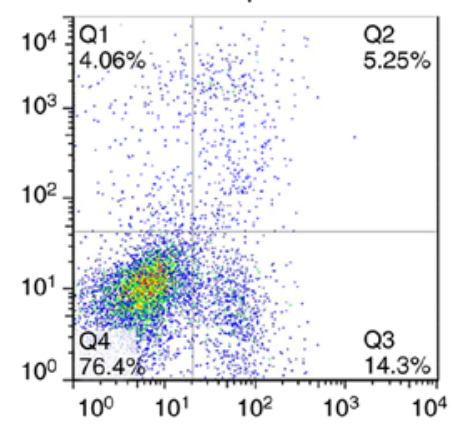

$50 \mu \mathrm{M}$

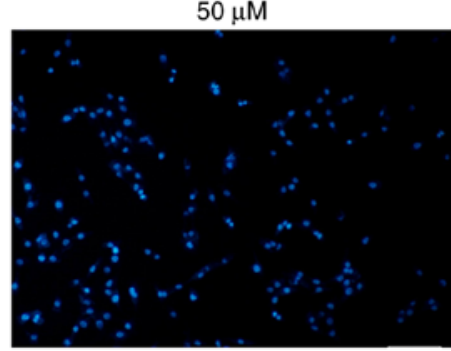

$50 \mu \mathrm{M}$

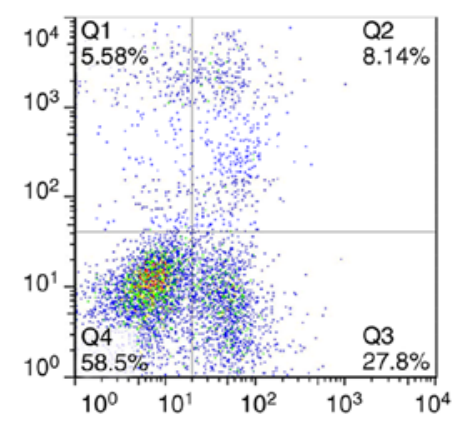

Annexin V

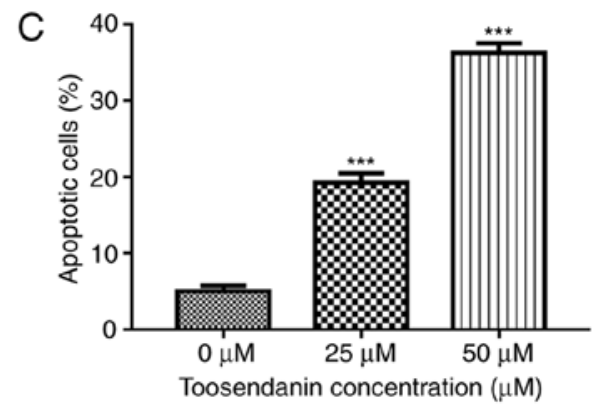

Figure 2. Toosendanin induces apoptosis and leads to changes in cell morphology. (A) Cells were incubated with 0,25 or $50 \mu \mathrm{M}$ toosendanin for $24 \mathrm{~h}$. Apoptotic nuclei stained with Hoechst 33258 (24 h) showed nuclear chromosome condensation and fragmentation (magnification, x200). (B) Representative images obtained by flow cytometry after double staining with Annexin V-FITC/PI. (C) Apoptosis rate (\%) of SK-ES-1 cells in the control and toosendanin treatment groups $(24 \mathrm{~h})$. Data are expressed as the mean $\pm \mathrm{SD}$ of three independent experiments. ${ }^{* * *} \mathrm{P}<0.001$ vs. the control.

apoptosis) was $5.03 \pm 0.71 \%$. After $24 \mathrm{~h}$ of treatment with 25 or $50 \mu \mathrm{M}$ toosendanin, the apoptotic rate increased to $19.32 \pm 1.26$ and $36.28 \pm 1.28 \%$, respectively (Fig. 2B). Thus, toosendanin induced apoptosis in a dose-dependent manner (Fig. 2C).
Toosendanin modulates the expression levels of caspase and $\mathrm{Bcl}-2$ family proteins. Western blot analysis was used to analyze expression levels of apoptosis-related protein Bcl-2, pro-apoptotic Bax, cytochrome $c$, caspase-3, -8 and -9 and 
A
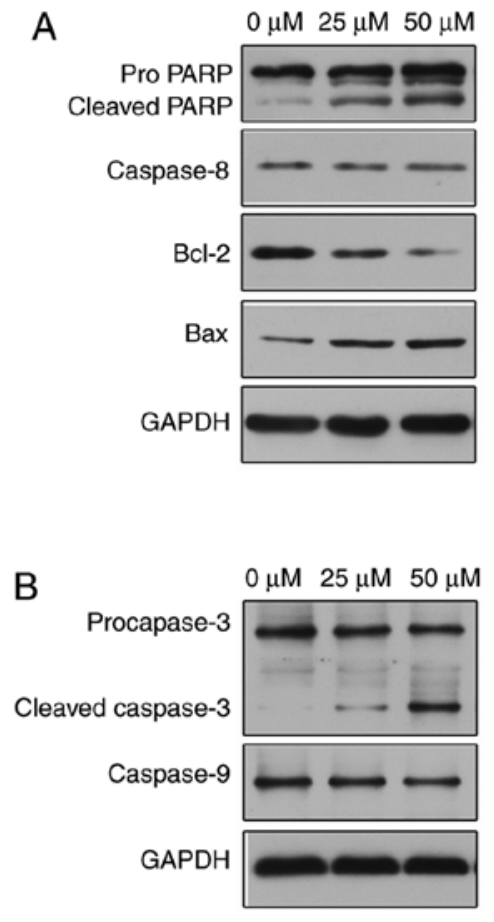
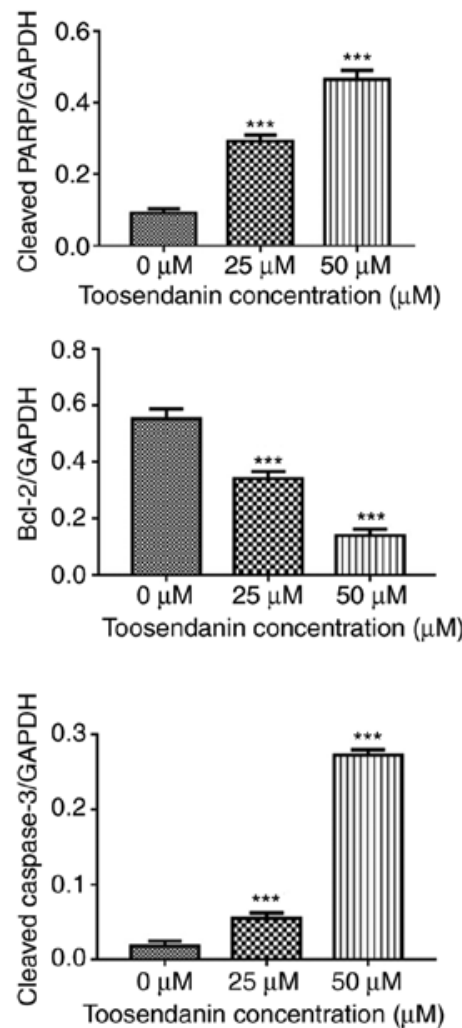
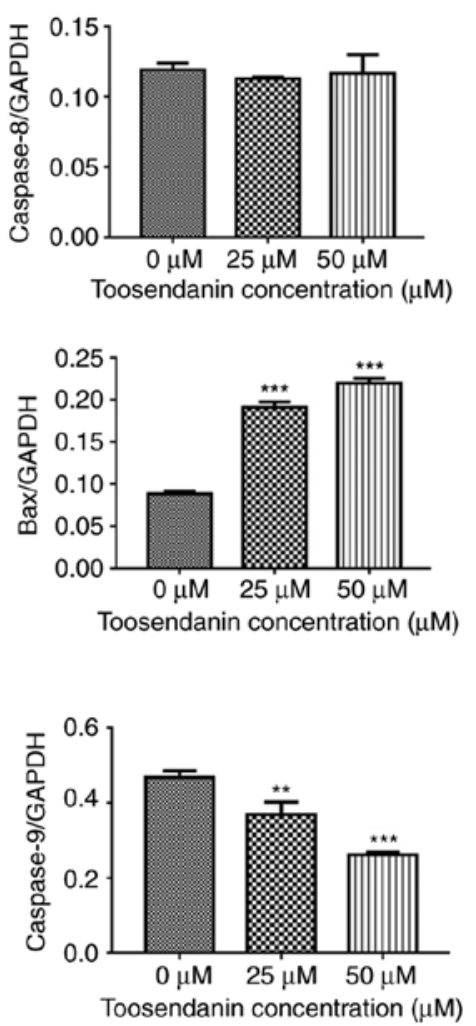
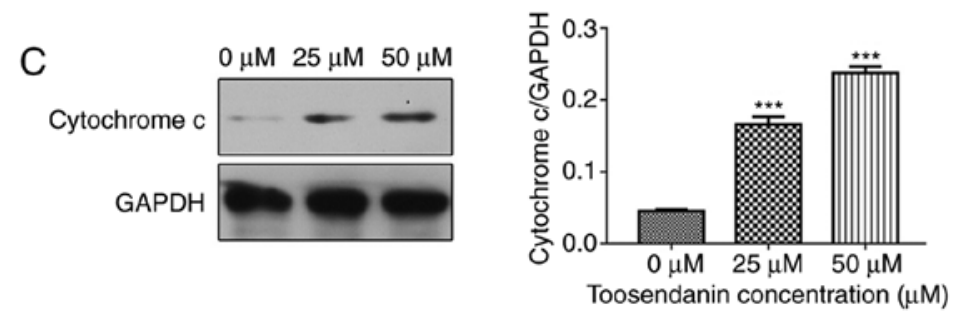

Figure 3. Effect of toosendanin on apoptosis-related protein levels. (A) The effect of toosendanin $(0,25$ or $50 \mu \mathrm{M})$ on protein expression levels of PARP, caspase-8, Bcl-2 and Bax in SK-ES-1 cells was analyzed by western blotting. Quantitative analysis was performed using ImageJ software and the data are expressed as the mean \pm SD of three independent experiments. ${ }^{* * *} \mathrm{P}<0.001$ vs. control. (B) Protein expression levels of caspase-3 and caspase-9 in SK-ES-1 cells were analyzed by western blotting after toosendanin $(0,25$ or $50 \mu \mathrm{M})$ treatment. Quantitative analysis was performed using ImageJ software and the data are expressed as the mean $\pm \mathrm{SD}$ of three independent experiments. ${ }^{* *} \mathrm{P}<0.01$ and ${ }^{* * * *} \mathrm{P}<0.001$ vs. the control. (C) Cytochrome c expression levels in SK-ES- 1 cells analyzed by western blotting after toosendanin $(0,25$ or $50 \mu \mathrm{M})$ treatment. Quantitative analysis was performed using ImageJ software and the data are expressed as the mean $\pm \mathrm{SD}$ of three independent experiments. ${ }^{* * * *} \mathrm{P}<0.001$ vs. the control. The results showed that toosendanin increased Bax protein levels and cytochrome c release as well as decreased Bcl-2 protein levels. Cleavage of caspase-3 and the key cellular substrate PARP were observed as well as downregulation of procaspase-3 and procaspase-9. Caspase-3 and caspase-9 were also cleaved and downregulated in a dose-dependent manner. Meanwhile, caspase- 8 expression levels did not change. These results indicate that toosendanin-induced apoptosis involves the caspase cascade and is triggered by the mitochondrial apoptotic pathway and that toosendanin activates the mitochondrial apoptotic pathway by modulating the expression of Bcl-2 family proteins. PARP, poly(ADP-ribose) polymerase; Bcl-2, B-cell lymphoma; Bax, Bcl-2-associated X protein.

PARP to identify the mechanism of toosendanin-induced SK-ES-1 apoptosis. The results showed that toosendanin increased Bax protein levels (Fig. 3A) and cytochrome $c$ release (Fig. 3C) as well as decreased Bcl-2 protein levels (Fig. 3A). This indicates that toosendanin activates the mitochondrial apoptotic pathway in SK-ES-1 cells by modulating the expression of Bcl-2 family proteins. At the same time, we evaluated the protein expression levels of caspase- $3,-8$ and -9 . Cleavage of caspase- 3 and the key cellular substrate PARP was observed as well as downregulation of procaspase- 3 and procaspase- 9 expression levels; caspase-3 and caspase- 9 were cleaved and downregulated in a dose-dependent manner (Fig. 3A and B). In contrast, expression levels of caspase- 8 did not change (Fig. 3A). These results indicate that toosendanin-induced apoptosis involves the caspase cascade and is triggered by the mitochondrial apoptotic pathway.

\section{Discussion}

As malignant tumors are the result of unchecked cell proliferation, inhibiting tumor cell proliferation and promoting tumor cell apoptosis are effective means to prevent tumor growth and eliminate tumors (15). Apoptosis, also known as programmed cell death, is the orderly death of cells controlled by genes that maintain internal environment stability. One of the characteristics of tumor cells is their ability to resist apoptosis (16) and thus, induction of tumor cell apoptosis is the mechanism of action of many antitumor drugs (17). With increasing attention 
on toosendanin, its antitumor effect has become a 'hot topic' in research. Tada et al (18) found that toosendanin exhibits strong cytotoxicity against human cancer cell lines (KB cells) and that the toxicity mechanism may be related to the C-14/C-15 epoxy structure of toosendanin. Zhang et al (19) reported that toosendanin may induce apoptosis in lymphoma U937 cells by arresting cells in $\mathrm{G}_{0} / \mathrm{G}_{1}$ and $\mathrm{S}$ phases. Furthermore, He et al (20) demonstrated that toosendanin possesses strong anticancer effects in vivo and in vitro via inducing mitochondria-dependent apoptosis in hepatocellular carcinoma cells. Meanwhile, Ju et al (21) discovered that the pro-apoptotic effects of toosendanin on human promyelocytic leukemia HL-60 cells were mediated through the c-Jun N-terminal kinase (JNK) signaling pathway. The above studies have shown that toosendanin is a potential antitumor drug with remarkable effects; however, the mechanism of action in regards to Ewing's sarcoma (ES) cells has not been elucidated. To this end, we employed human SK-ES-1 cells to explore the mechanism of action of toosendanin in regards to ES.

The present study found that toosendanin has an inhibitory effect on the proliferation of the SK-ES-1 cell line in a timeand dose-dependent manner. These findings are consistent with the literature reported on other types of tumors, which also have identified toosendanin as an anticancer agent. To the best of our knowledge, this is the first study to explore the effects of toosendanin on ES in vitro.

To further explore the mechanism of apoptosis induction in SK-ES-1 cells by toosendanin, the changes in expression levels of caspase-3, -8 and -9 , and other apoptosis-related genes were determined by western blotting. There are two main pathways of apoptosis, the mitochondrial pathway and the death receptor pathway. The mitochondrial pathway involves the ratio of pro-apoptotic protein Bax to anti-apoptotic protein $\mathrm{Bcl}-2$, which affects the release of many apoptotic proteins in the mitochondrial membrane space, such as cytochrome $c$. Cytochrome $c$ activates caspase- 9 and reactivates caspase-3. The Fas/tumor necrosis factor (TNF) receptor located in the cell membrane of the death receptor pathway is activated by apoptosis, which activates caspase- 8 that goes on to activate caspase-3. Subsequently, activated caspase- 3 causes cleavage or degradation of key cellular substrates including PARP, resulting in cell morphological changes, DNA double-strand breaks, and other characteristics of apoptotic cells $(22,23)$. In the present study, toosendanin-induced apoptosis was accompanied by a change in the ratio of $\mathrm{Bax} / \mathrm{Bcl}-2$, which activated caspase- 9 and caspase-3. In addition, PARP cleavage was also observed. These findings indicate that SK-ES-1 cell apoptosis induced by toosendanin is triggered by the mitochondrial pathway.

In conclusion, toosendanin was found to inhibit ES cell viability and apoptosis through the mitochondrial apoptosis pathway. Our findings support the utilization of toosendanin as a potential therapeutic agent for the treatment of ES. However, the present study has certain limitations. First, we only employed in vitro experiments and thus further research is needed to elucidate the in vivo effects of toosendanin on SK-ES-1 xenograft tumors in nude mice. Second, we cannot rule out whether toosendanin participates in other signaling pathways in the induction of ES cell apoptosis. Furthermore, safety must be guaranteed before clinical application of 'natural medicine'. Previous studies have reported that high doses of toosendanin $(80 \mathrm{mg} / \mathrm{kg})$ result in serious liver injury in mice (24), while the effect of toosendanin on normal or healthy cells in vitro remains unclear. Our in vitro experiments demonstrated that the $\mathrm{IC}_{50}$ value of toosendanin in SK-ES-1 cells was $32.95 \mu \mathrm{M}$, and the cytotoxicity of this concentration must be evaluated prior to use. Thus, future research must comprehensively evaluate the effects of toosendanin on normal cells through in vivo and in vitro experiments to understand the therapeutic range of toosendanin.

\section{Acknowledgements}

Not applicable.

\section{Funding}

The present study was supported by the Jiangxi Provincial Department of Science and Technology (grant no. 20171ACG70006).

\section{Availability of data and materials}

The datasets used and/or analyzed during the present study are available from the corresponding author on reasonable request.

\section{Authors' contributions}

TG, AX, BZ and MD conceived and designed the study; TG, AX and XL performed the experiments; BZ and HZ analyzed the data; TG, BZ and MD wrote the paper; XL and JZ prepared the figures; TG, AX, XL, HZ, BZ and MD reviewed and edited the manuscript. All authors read and approved the manuscript and agree to be accountable for all aspects of the research in ensuring that the accuracy or integrity of any part of the work are appropriately investigated and resolved.

\section{Ethics approval and consent to participate}

Not applicable.

\section{Patient consent for publication}

Not applicable.

\section{Competing interests}

The authors declare that they have no competing interests.

\section{References}

1. Ahmed S, Anuntiyo J, Malemud CJ and Haqqi TM: Biological basis for the use of botanicals in osteoarthritis and rheumatoid arthritis: A review. Evid Based Complement Alternat Med 2: 301-308, 2005.

2. Zhang K, Wang X, Wang C, Zheng H, Li T, Xiao S, Wang M, Fei C, Zhang L and Xue F: Investigation of quinocetone-induced mitochondrial damage and apoptosis in HepG2 cells and compared with its metabolites. Environ Toxicol Pharmacol 39: 555-567, 2015.

3. Zhang J, Song J, Wu D, Wang J and Dong W: Hesperetin induces the apoptosis of hepatocellular carcinoma cells via mitochondrial pathway mediated by the increased intracellular reactive oxygen species, ATP and calcium. Med Oncol 32: 101, 2015. 
4. Pieme CA, Santosh GK, Tekwu EM, Askun T, Aydeniz H, Ngogang JY, Bhushan S and Saxena AK: Fruits and barks extracts of Zanthozyllum heitzii a spice from Cameroon induce mitochondrial dependent apoptosis and G0/G1 phase arrest in human leukemia HL-60 cells. Biol Res 47: 54, 2014.

5. Zhou Q, Wu X, Wen C, Wang H, Wang H, Liu H and Peng J: Toosendanin induces caspase-dependent apoptosis through the p38 MAPK pathway in human gastric cancer cells. Biochem Biophys Res Commun 505: 261-266, 2018.

6. Shi YL and Li MF: Biological effects of toosendanin, a triterpenoid extracted from Chinese traditional medicine. Prog Neurobiol 82: 1-10, 2007.

7. Pei Z, Fu W and Wang G: A natural product toosendanin inhibits epithelial-mesenchymal transition and tumor growth in pancreatic cancer via deactivating Akt/mTOR signaling. Biochem Biophys Res Commun 493: 455-460, 2017.

8. Zhang T, Li J, Yin F, Lin B, Wang Z, Xu J, Wang H, Zuo D Wang G, Hua Y and Cai Z: Toosendanin demonstrates promising antitumor efficacy in osteosarcoma by targeting STAT3. Oncogene 36: 6627-6639, 2017.

9. Windsor R, Strauss S, Seddon B and Whelan J: Experimental therapies in Ewing's sarcoma. Expert Opin Investig Drugs 18 143-159, 2009.

10. Teicher BA, Bagley RG, Rouleau C, Kruger A, Ren Y and Kurtzberg L: Characteristics of human Ewing/PNET sarcoma models. Ann Saudi Med 31: 174-182, 2011.

11. Zhang Z, Huang L, Yu Z, Chen X, Yang D, Zhan P, Dai M, Huang S, Han $Z$ and Cao K: Let-7a functions as a tumor suppressor in Ewing's sarcoma cell lines partly by targeting cyclin-dependent kinase 6. DNA Cell Biol 33: 136-147, 2014

12. Ross KA, Smyth NA, Murawski CD and Kennedy JG: The biology of ewing sarcoma. ISRN Oncol 2013: 759725, 2013.

13. Scotlandi K: Targeted therapies in Ewing's sarcoma. Adv Exp Med Biol 587: 13-22, 2006.

14. Ye C, Yu X, Zeng J, Dai M and Zhang B: Effects of baicalein on proliferation, apoptosis, migration and invasion of Ewing's sarcoma cells. Int J Oncol 51: 1785-1792, 2017.
15. Cohen GM: Caspases: The executioners of apoptosis. Biochem J 326: 1-16, 1997.

16. Singhal S, Vachani A, Antin-Ozerkis D, Kaiser LR and Albelda SM: Prognostic implications of cell cycle, apoptosis, and angiogenesis biomarkers in non-small cell lung cancer: A review. Clin Cancer Res 11: 3974-3986, 2005.

17. Viktorsson K, Lewensohn R and Zhivotovsky B: Apoptotic pathways and therapy resistance in human malignancies. Adv Cancer Res 94: 143, 2005.

18. Tada K, Takido M and Kitanaka S: Limonoids from fruit of Melia toosendan and their cytotoxic activity. Phytochemistry 51: 787-791, 1999.

19. Zhang B, Wang ZF, Tang MZ and Shi YL: Growth inhibition and apoptosis-induced effect on human cancer cells of toosendanin, a triterpenoid derivative from chinese traditional medicine. Invest New Drugs 23: 547-553, 2005.

20. He Y, Wang J, Liu X, Zhang L, Yi G, Li C, He X, Wang P and Jiang $\mathrm{H}$ : Toosendanin inhibits hepatocellular carcinoma cells by inducing mitochondria-dependent apoptosis. Planta Med 76: 1447-1453, 2010.

21. Ju J, Qi Z, Cai X, Cao P, Liu N, Wang S and Chen Y: Toosendanin induces apoptosis through suppression of JNK signaling pathway in HL-60 cells. Toxicol In Vitro 27: 232-238, 2013.

22. Wang X: The expanding role of mitochondria in apoptosis. Genes Dev 15: 2922-2933, 2001.

23. Wang $\mathrm{C}$ and Youle RJ: The role of mitochondria in apoptosis*. Annu Rev Genet 43: 95-118, 2009.

24. Lu X, Ji C, Tong W, Lian X, Wu Y, Fan X and Gao Y: Integrated analysis of microRNA and mRNA expression profiles highlights the complex and dynamic behavior of toosendanin-induced liver injury in mice. Sci Rep 6: 34225, 2016.

(i) (3) This work is licensed under a Creative Commons Attribution-NonCommercial-NoDerivatives 4.0 International (CC BY-NC-ND 4.0) License. 